\title{
A 10-year clinical outcome of radiotherapy as an adjuvant or definitive treatment for primary tracheal adenoid cystic carcinoma

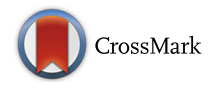

\author{
Hyoung Uk Je', Si Yeol Song ${ }^{2^{*}}$ (D), Dong Kwan Kim³ ${ }^{3}$, Yong-Hee Kim³ ${ }^{3}$ Seong-Yun Jeong ${ }^{4}$, Geum Mun Back ${ }^{2,5}$, \\ Wonsik Choi ${ }^{6}$, Su Ssan Kim², Seung-ll Park ${ }^{3}$ and Eun Kyung Choi ${ }^{2^{*}}$
}

\begin{abstract}
Background: To evaluate the role of radiotherapy (RT) as an adjuvant or definitive treatment in primary tracheal adenoid cystic carcinoma (ACC) for local tumor control and survival.

Methods: A retrospective chart review was performed in 22 patients treated with adjuvant or definitive RT for primary tracheal ACC at a single center between November 1994 and December 2008.

Results: Thirteen and 9 patients received adjuvant and definitive RT, respectively. Microscopic residual disease after surgery was pathologically reported in 11 patients. The median RT dose was 59.4 Gy for adjuvant and 74.4 Gy for definitive RT. The overall response rate for definitive RT was 77.8\%. Six patients in the definitive RT group exhibited local progression (LP), whereas 14 patients in both groups exhibited distant metastasis. The most common recurrence site in cases of treatment failure was the lung parenchyma. The median follow-up duration was 123 months, and the 10-year overall survival (OS) rate was 54.2\%. Although LP was the most common cause of death (4 patients), two-thirds of the patients treated with definitive RT lived for $>5$ years. The 5 -year and 10-year LP-free survival (LPFS) rates in the definitive RT group were 66.7 and $26.7 \%$, respectively. Patients with higher RT dose by brachytherapy boost had good 5-year OS, 83.3\%, and showed no local progression till 5-years. Most of the RT-induced side-effects were mild and tolerable, but 2 patients died of tracheal stenosis without any tumor recurrence.

Conclusions: Adjuvant RT may be suitable for controlling microscopic residual disease, whereas definitive RT may yield appropriate long-term survival in $>50 \%$ patients with unresectable tracheal ACC. Dose escalation should be considered to warrant long-term survival in definitive RT.
\end{abstract}

Keywords: Tracheal cancer, Adenoid cystic carcinoma, ACC, Radiotherapy, Unresectable, Survival

\section{Background}

Tracheal cancer is a rare neoplasm that accounts for only $2 \%$ of upper airway tumors [1]. The incidence of primary tracheal tumors is $<0.2$ per 100,000 persons per year in the United States [2]. Squamous cell carcinoma (SCC) is the most common histological type of tracheal malignancy, followed by adenoid cystic carcinoma (ACC) [3, 4]. Tracheal ACC was first reported by Morgagni in 1762. ACC of the trachea and bronchi arises from glands in the

\footnotetext{
*Correspondence: siyeol.song@gmail.com; coocoori@amc.seoul.kr; ekchoi@amc.seoul.kr

${ }^{2}$ Department of Radiation Oncology, Asan Medical Center, University of Ulsan College of Medicine, 88, Olympic-ro 43-gil, Songpa-gu, Seoul 05505, South Korea

Full list of author information is available at the end of the article
}

tracheobronchial mucosa. ACC is characterized by slow growth, and distant metastasis has been reported as late as 25 years after diagnosis [5-7].

Surgery is considered as the treatment of choice for ACC, and most articles have focused on the surgical outcome. Radiotherapy (RT) was traditionally used as an adjuvant treatment for controlling microscopic disease or as a salvage treatment for unresectable disease; however, the exact role of RT remains unclear due to the rarity of such reports. Although a few studies have described the use of surgery followed by adjuvant therapy, only a few factors related to RT planning or dose have been described [7-9]. In particular, for definitive RT, additional information should be provided, as most retrospective 
studies thus far have analyzed a very small number of patients, have used a classical RT technique or irradiated a relatively lower RT dose $[6,8,10,11]$. The most recently available data on modern RT techniques are described in case reports, and these provide promising results for RT of ACC of the trachea [12-14].

In the present study, we aimed to evaluate the role of RT with modern techniques as adjuvant treatment after surgical resection or as definitive treatment in inoperable settings for primary tracheal ACC for local tumor control and survival.

\section{Methods}

Between November 1994 and December 2008, a total of 25 patients were treated with RT for pathologically confirmed primary ACC of the cervical or upper thoracic airway in our institution. We retrospectively reviewed the medical records and the study was approved by the institutional review board of Asan Medical Center, Seoul, Korea (AMCIRB, 2012-0470). Tumor location was assessed by using a bronchoscope or computed tomography $(\mathrm{CT})$, and cases with tumors located in the trachea and carina with/without main bronchus involvement were included in the study. The epicenters of primary tumor in all patients were trachea. We excluded patients with a distant metastasis prior to treatment. However, 1 patient with single lung metastasis at the initial diagnosis was included in this study, because the metastatic lesion had been successfully treated with stereotactic body radiotherapy and complete remission was achieved in that case.

The tracheal tumor stage was determined according to the staging system for primary tracheal malignancies proposed by Bhattacharyya et al [4]. We measured tumor size by using a fiber-optic bronchoscope or CT scan.

The eligibility for surgical excision was determined via a multidisciplinary conference or clinic, after reviewing the results of diagnostic studies and the patient's condition. A dedicated thoracic surgeon determined the surgical approach and extent.

Adjuvant RT was usually initiated 4-6 weeks after surgery, and the standard dose was 50.4 Gy for 28 fractions. A boost dose was provided if the tumor had a surgical margin. Approximately 60-66 Gy of conventional fractionation was the standard dose for definitive RT, although brachytherapy (BT) was occasionally adopted for a local boost. In all cases of RT, a linear accelerator with threedimensional conformal RT or intensity-modulated RT (IMRT) was used, and an iridium-192 high-dose BT system was employed. BT was conducted at $1 \mathrm{~cm}$ from the iridium source, and a boost dose of 15 Gy or 21 Gy with 5-7 Gy per fraction was used. The initial irradiation field encompassed the tumor or tumor bed as well as the adjacent mediastinal or supraclavicular lymph nodes in most patients. Clinical target volume (CTV) was usually expanded $3 \mathrm{~cm}$ in longitudinal from tumor bed or primary tumor and adjacent lymph node stations were included it at designated level. Planning target volume (PTV) was generally expanded $1 \mathrm{~cm}$ in both longitudinal and axial from the CTV. Dose constraints for organ at risk (OAR) like esophagus, lung or other organs were not absolutely determined in this study, while we tried not to produce hot-spot at esophagus due to the proximity to PTV and institutional dose constraint for whole lung was V $20<30 \%$.

In the definitive RT group, we judged tumor response according to the Response Evaluation Criteria in Solid Tumors (RECIST) criteria [15]. Response evaluation and routine follow-up was performed by $\mathrm{CT}$ images and bronchoscopy with/without biopsy was added to confirm residual tumor after definitive RT. Positron emission tomography (PET) was adopted in only a half patient, because it was not introduced in the early phase of this enrollment. PET was additionally used to confirm local progression or distant metastasis in some cases, but not routinely acquired. The initial response of RT was assessed at 1 or 2 months after RT. We used the Radiation Therapy Oncology Group (RTOG) toxicity grading system to score acute and chronic complications. Patients visited the outpatient clinic at 3-month intervals during the initial 2 years and at 6-month intervals thereafter.

The survival rate was calculated using the KaplanMeier survival analysis method. Survival curves were compared using the log-rank test and odds ratio from cox-regression analysis was calculated for multivariate analysis. All analyses were based on a two-sided test for significance (0.05), and were conducted using the SPSS Statistics 21.0 software (SPSS Inc., Chicago, IL).

\section{Results}

\section{Patient and treatment characteristics}

A total of 22 patients were analyzed; three patients were excluded due to coincident multiple lung metastasis or because they were lost to follow-up immediately after treatment, with no evidence of progression or toxicity. Patient characteristics are presented in Table 1. Fourteen (63.6\%) patients were female. The most common presenting symptom was dyspnea. In many cases, symptoms of airway obstruction, such as dyspnea or wheezing, led to a misdiagnosis of asthma, which delayed the diagnosis of ACC.

The treatment characteristics are summarized in Table 2. Thirteen patients underwent surgery, and the resection margin status was $\mathrm{R} 1$ in 11 patients (84.6\%). These 13 patients received adjuvant RT and the other 9 patients received definitive RT. The fraction size of external beam radiotherapy (EBRT) ranged from $1.8 \mathrm{~Gy}$ to $2.2 \mathrm{~Gy}$, and 3 or 4 sessions of 5-7 Gy BT were conducted for a local boost after EBRT in some cases. 
Table 1 Patient characteristics

\begin{tabular}{|c|c|c|}
\hline & Number of & ths (\%) \\
\hline & PORT & Definitive RT \\
\hline Total number of patients & 13 & 9 \\
\hline Median age, years (range) & $40(27-69)$ & $44(37-68)$ \\
\hline Gender & & \\
\hline Male & $3(23)$ & $5(56)$ \\
\hline Female & $10(67)$ & $4(44)$ \\
\hline Initial presenting symptom & & \\
\hline Dyspnea & $9(69)$ & $7(78)$ \\
\hline Cough & $1(8)$ & $2(22)$ \\
\hline Hoarseness & $2(15)$ & \\
\hline None & $1(8)$ & \\
\hline Location of the tumor & & \\
\hline Upper half, cervical & $5(38)$ & $3(33)$ \\
\hline Lower half, thoracic & $3(24)$ & $4(45)$ \\
\hline Carina involving main bronchus & $5(38)$ & $2(22)$ \\
\hline Clinical T stage & & \\
\hline $\mathrm{T} 1$ & $6(46)$ & \\
\hline $\mathrm{T} 2$ & $7(54)$ & $8(89)$ \\
\hline T4 & & $1(11)$ \\
\hline Clinical N stage & & \\
\hline NO & $13(100)$ & $9(100)$ \\
\hline Clinical M stage & & \\
\hline MO & $13(100)$ & $8(89)$ \\
\hline M1 & & $1(11)$ \\
\hline
\end{tabular}

PORT post-operative radiotherapy, $R T$ radiotherapy

Two patients in definitive RT group received IMRT without BT boost. Only 1 patient received concurrent chemotherapy with gemcitabine plus cisplatin.

\section{Tumor response and pattern of failure}

One patient exhibited complete remission (CR), and showed only fibrotic residual tissue on biopsy, after definitive RT. None of the patients exhibited progressive disease during the initial response evaluation. The overall response rate, including CR or partial remission (PR), in 9 patients with an inoperable tracheal tumor was 77.8\% (Table 3).

Six $(27.3 \%)$ patients developed local progression (LP) during the follow-up period, although LP developed at the initial nidus only in 3 cases. LP developed only in patients treated with definitive RT without surgery. LP was pathologically confirmed in 3 patients experiencing early LP, while progression in the other patients was judged by gradual tumor enlargement on serial CT image. A total of 14 (63.6\%) patients developed distant metastasis, and the most common site was lung parenchyma. The mean duration to lung metastasis in 12 patients was 88.3 months. According
Table 2 Treatment characteristics

\begin{tabular}{|c|c|}
\hline & No. (\%) \\
\hline Surgery & 13 \\
\hline \multicolumn{2}{|l|}{ Type } \\
\hline Tracheal resection & $6(46.2)$ \\
\hline Laryngotracheal resection & $1(7.7)$ \\
\hline Bronchial resection & $1(7.7)$ \\
\hline Pneumonectomy & $4(30.7)$ \\
\hline Endobronchial resection & $1(7.7)$ \\
\hline \multicolumn{2}{|l|}{ Resection margin status } \\
\hline Ro & $2(15.4)$ \\
\hline R1 & $11(84.6)$ \\
\hline Radiotherapy & 22 \\
\hline \multicolumn{2}{|l|}{ Type } \\
\hline PORT & $13(59.1)$ \\
\hline Definitive RT & $8(36.4)$ \\
\hline Definitive CCRT & $1(4.5)$ \\
\hline \multicolumn{2}{|l|}{ Technique } \\
\hline EBRT alone & $16(72.7)$ \\
\hline EBRT and brachytherapy boost & $6(27.3)$ \\
\hline Median dose (Gy) & 59.8 (range, 50.4-80) \\
\hline PORT & 59.4 (range, 50.4-66) \\
\hline Definitive RT & 74.4 (range, 66-80) \\
\hline \multicolumn{2}{|l|}{ Radiotherapy field } \\
\hline Trachea only & $1(4.5)$ \\
\hline Trachea with regional lymph node & $21(95.5)$ \\
\hline
\end{tabular}

to the first site of failure, the main pattern of failure was distant metastasis (Table 4).

\section{Survival}

The median follow-up duration was 123 months (10.2 years; range, 13-232 months). The 5-year and 10-year overall survival (OS) rates were 81.8 and $54.2 \%$, respectively. Fifteen $(68.2 \%)$ patients died during the follow-up period. The cause of death was LP in 4, lung metastasis in 2, peritoneal seeding in 2, other metastasis in 3 , tracheal stenosis in 2 , and no relation to the disease in 2 cases. The 5 -year and 10-year disease-free survival (DFS) rates were 47.8 and $28.7 \%$, respectively. None of the patients developed LP or distant metastasis after 10 years. The 5 -year LP-free survival (LPFS) rate was $100 \%$ in the postoperative RT (PORT) group and $66.7 \%$ in the definitive RT group $(p<0.01)$. Three patients developed LP over 5 years after RT, and the 10-year LPFS rate decreased to $26.7 \%$ after definitive $\mathrm{RT}$. The mean LPFS in all 22 patients was 122 months, whereas that in the definitive RT group was 57 months (Table 4). 
Table 3 Details of the patients who were treated with definitive radiotherapy

\begin{tabular}{|c|c|c|c|c|c|c|c|c|c|c|c|c|}
\hline Sex & Age & $T$ & Size $(\mathrm{cm})$ & RT dose & BT boost & RESP & $L P$ & $\begin{array}{l}\text { RT- LP } \\
\text { (mo) }\end{array}$ & $\mathrm{DM}$ & RT-DM (mo) & Cause of Death & $\begin{array}{l}\text { OS } \\
(\mathrm{mo})\end{array}$ \\
\hline$F$ & 61 & 2 & 4 & 70.8 & + & $C R$ & - & & - & & Stenosis & 172 \\
\hline M & 48 & 2 & 6 & 70.2 & & $P R$ & + & 36 & - & & LP & 107 \\
\hline M & 44 & 2 & 6 & 79.8 & + & $P R$ & + & 104 & + & 28 & LP & 107 \\
\hline M & 68 & 4 & 5 & 74.4 & + & $P R$ & - & & + & 18 & DM & 51 \\
\hline M & 36 & 2 & 4 & 74.4 & + & $P R$ & - & & + & 85 & Other & 232 \\
\hline M & 37 & 2 & 6 & 80.0 & + & PR & + & 77 & + & 24 & DM & 115 \\
\hline$F$ & 37 & 2 & 5 & 79.8 & + & SD & + & 87 & + & 29 & LP & 87 \\
\hline$F$ & 66 & 2 & 7 & 66.0 & & SD & + & 17 & + & 17 & LP & 32 \\
\hline $\mathrm{F}$ & 43 & 2 & 7 & 66.0 & & $P R$ & + & 21 & + & 21 & $\mathrm{DM}$ & 26 \\
\hline
\end{tabular}

$T$ T-stage, $R T$ radiotherapy, $B T$ brachytherapy, $R E S P$ initial response after $R T, L P$ local progression, $D M$ distant metastasis, $R T$ - $L P$ time interval from $R T$ to $L P, R T$-DM time interval from RT to DM, OS overall survival, mo months

\section{Prognostic factor}

Initial local tumor stage (T1 vs. T2-4) was a unique prognostic factor for OS $(p=0.045)$, whereas surgery had a significance for LPFS $(p<0.01)$ in univariate analysis. Surgery tended to prolong OS, but it couldn't reach statistical significance $(p=0.058)$ However, no factor could reach statistical significance for OS or LPFS in multivariate analysis, but odds ratio of PORT for LPFS was very low value, 0.005 , which might mean the decreased risk of local progression (Table 5). Gender or location of primary tumor had no effect on treatment results. In sub-analysis with only definitive RT group, intensified RT dose with BT boost was a statistically significant factor for OS $(p=0.036)$ and LFPS $(p=$ 0.002) (Table 6) (Fig. 1).

Table 4 Treatment results for all patients

\begin{tabular}{lllll}
\hline & All patients & PORT & Definitive RT & $p$ value \\
\hline No. of patients & 22 & 13 & 9 & \\
Local recurrence & $6(27 \%)$ & 0 & $6(67 \%)$ & \\
Distant metastasis & $14(64 \%)$ & $7(54 \%)$ & $7(78 \%)$ & \\
First site of recurrence & & & & \\
Local & $1(5 \%)$ & 0 & $1(11 \%)$ & \\
Distant & $12(55 \%)$ & $7(54 \%)$ & $5(56 \%)$ & \\
Local \& distant & $2(9 \%)$ & 0 & $2(22 \%)$ & \\
5-yr. LPFS (\%) & 85.7 & 100.0 & 66.7 & $<.01$ \\
10-yr. LPFS (\%) & 70.2 & 100.0 & 26.7 & \\
5-yr. DMFS (\%) & 52.6 & 67.7 & 33.3 & .209 \\
10-yr. DMFS (\%) & 33.5 & 42.3 & 22.2 & \\
5-yr. DFS (\%) & 47.8 & 67.7 & 22.2 & .054 \\
10-yr. DFS (\%) & 28.7 & 42.3 & 11.1 & \\
5-yr. OS (\%) & 81.8 & 92.3 & 66.7 & .058 \\
10-yr. OS (\%) & 54.2 & 76.9 & 22.2 & \\
\hline
\end{tabular}

$R T$ radiotherapy, PORT post-operative radiotherapy, LPFS local progression free survival, DFS disease free survival, OS overall survival

\section{Treatment-related complications}

The most frequent complaint during RT was odynophagia, although the condition could be tolerated without narcotic analgesics in most patients. Three patients developed grade 3 esophagitis during RT, but there was no esophageal stenosis after treatment. Nine patients developed grade 2 or lower radiation-induced lung fibrosis.

The major concern after treatment was tracheal stenosis (2 patients). Tracheal stenosis without any evidence of recurrence developed 1 year after definitive RT in 1 case. This patient lived for 13 years after the diagnosis of stenosis, but needed the implantation of a tracheal stent. She finally died of tracheal infection around the stent. Another patient received PORT after surgery with permanent tracheostomy. She complained of dyspnea at 2 months after RT, but did not exhibit any recurrence. Nevertheless, she suffered from the narrowing of the tracheostomy site due to the gradual increase in granulation tissue, and died of abrupt respiratory failure after 1 year.

\section{Discussion}

Adenoid cystic carcinoma originally arises from the salivary glands and is a slowly progressing malignancy, with late metastasis to the lung, bone, and brain. The most common site in the airway is the trachea and the prognosis for ACC is better than that for SCC tracheal cancer [16, 17]. A few retrospective studies on the outcome of RT for tracheal ACC are summarized in Table 7 . The studies mostly reported good 5-year OS of $>70 \%$; however, the results could not be directly compared with those of the current study, due to the lack of a definite consensus about the staging system for tracheal tumor and the difference in the treatment modalities.

In the present study, local recurrence was not observed in patients treated with PORT after surgical resection over a long follow-up duration, despite residual 
Table 5 Prognostic factors for survival; univariate and multivariate analysis

\begin{tabular}{|c|c|c|c|c|c|c|c|c|c|}
\hline & \multirow[t]{3}{*}{ Description } & \multirow{2}{*}{\multicolumn{2}{|c|}{$\frac{\text { Uni-variate }}{p \text {-value }}$}} & \multicolumn{6}{|c|}{ Multi-variate } \\
\hline & & & & \multicolumn{2}{|c|}{ Odds ratio } & \multicolumn{2}{|l|}{$95 \% \mathrm{Cl}$} & \multicolumn{2}{|c|}{$p$-value } \\
\hline & & OS & LPFS & OS & LPFS & OS & LPFS & $\mathrm{OS}$ & LPFS \\
\hline Gender & Male vs. Female & .455 & .511 & & & & & & \\
\hline Location & Cervical vs. Thoracic & .910 & .986 & & & & & & \\
\hline T stage & T1 vs. T2-4 & .045 & .075 & 3.144 & 1.014 & $0.566-17.469$ & * & .190 & .999 \\
\hline Surgery & Definitive RT vs. PORT & .058 & .001 & 0.628 & 0.005 & $0.185-2.132$ & * & .456 & .338 \\
\hline
\end{tabular}

Cl confidence interval, OS overall survival, LPFS local-progression free survival, PORT post-operative RT, * two wide range: 0 -maximal

primary nidus after $\mathrm{R} 1$ resection in 11 (84.6\%) patients. This clearly supports the role of RT for controlling microscopic disease in tracheal ACC. In contrast, there were $6(66.7 \%)$ cases of LP in the definitive RT group. Hence, surgical resection of the tumor may be important for local tumor control, even though the tracheal tumor cannot be completely removed.

Nevertheless, definitive RT remains important in unresectable patients. Two-thirds of our study patients in definitive group lived for $>5$ years after RT, and the 10 -year OS was $22.2 \%$. Considering that these cases had relatively locally advanced tumors (T2 in 8 and T4 in 1 patient), this result does not appear to be disappointing, and hence, RT could be considered as an effective modality for unresectable tracheal ACC. Three patients developed LP more than 5 years after RT, which indicates that long-term evaluation with an appropriate imaging study should be recommended in these cases.

There is an issue in appropriate RT dose for unresectable tracheal ACC, but there is no consensus. In this study, patients treated with higher RT dose via BT boost had better long-term local tumor control and survival than those of patients receiving relatively lower RT dose. A 5 yr.-OS in patients treated with higher RT dose was very high, 88.3\%. A 5 yr.-local control was $100 \%$ in 6 patients with higher RT dose, while all 3 patients with lower RT dose suffered from LP within 5 years after treatment (Fig. 1). Although this result was obtained from small number of patients $(n=9)$, it is sufficient to explain the necessity

Table 6 Radiotherapy dose and survival in definitive RT $(n=9)$

\begin{tabular}{llll}
\hline & $\begin{array}{l}\text { EBRT alone (low- } \\
\text { dose) }\end{array}$ & $\begin{array}{l}\text { EBRT + BT boost (high- } \\
\text { dose) }\end{array}$ & $\begin{array}{l}p \text { - } \\
\text { value }\end{array}$ \\
\hline $\begin{array}{l}\text { No. of } \\
\text { patients }\end{array}$ & 3 & 6 & \\
RT dose (Gy) & & & \\
$\quad$ Median & 66.0 & 77.1 & \\
Range & $66.0-70.2$ & $70.8-80.0$ & .002 \\
5-yr. LPFS (\%) & 0.0 & 100.0 & .036 \\
\hline 5-yr. OS (\%) & 33.3 & 83.3 & \\
\hline
\end{tabular}

$R T$ : radiotherapy, EBRT external-beam $R T, B T$ brachytherapy, LPFS localprogression free survival, $O S$ overall survival of escalated RT dose to sterilize gross tracheal ACC. We expect the IMRT can be a troubleshooter for escalating RT dose without additional treatment-related side effect, even if we used BT boost in most patients of this study that was done in period prior to clinical application of IMRT.

About 50\% of ACC patients might eventually develop distant metastases, although regional lymph node involvement is reported to have been rare in several cohorts $[6,18]$. In our present study, the main pattern of failure was distant metastasis. No regional lymph node metastasis was observed before or after treatment in our cases, but distant metastasis occurred in $63.6 \%$ of patients; the lung was the main metastatic site. The time interval between treatment and distant metastasis ranged from 8.4 to 91 months. Although a patient may develop a distant metastasis, they could remain alive with the metastasis for a long duration (up to 232 months); this may be related to the slow progression of ACC. The mean time interval from DM to death was 78 months, whereas the mean time interval between LP and death was 23 months in our series. This finding suggests that active local control can increase survival, although there is a risk of minimal distant metastasis in patients with tracheal ACC.

More than half of the patients in our present study lived for $>5$ years after definitive RT. Although this survival rate is inferior to that of the PORT group, it is sufficient to offer a feasible solution for patients with unresectable tracheal ACC. Nevertheless, LP developed between 5 years and 10 years after RT in 3 cases, and hence, the presence of symptoms in the airway should be monitored and proper imaging studies should be conducted for at least 10 years. The 10-year LPFS rate decreased to $26.7 \%$ in the present study cohort, and none of our patients exhibited LP after 10 years.

After resection with/without RT for tracheal cancer, some patients have been reported to develop severe complications such as recurrent laryngeal nerve palsy, tracheal stenosis, dysphagia, and airway granulation [6, 19-21]. In the present study, 2 patients developed tracheal stenosis, which resulted in death. Hence, the 


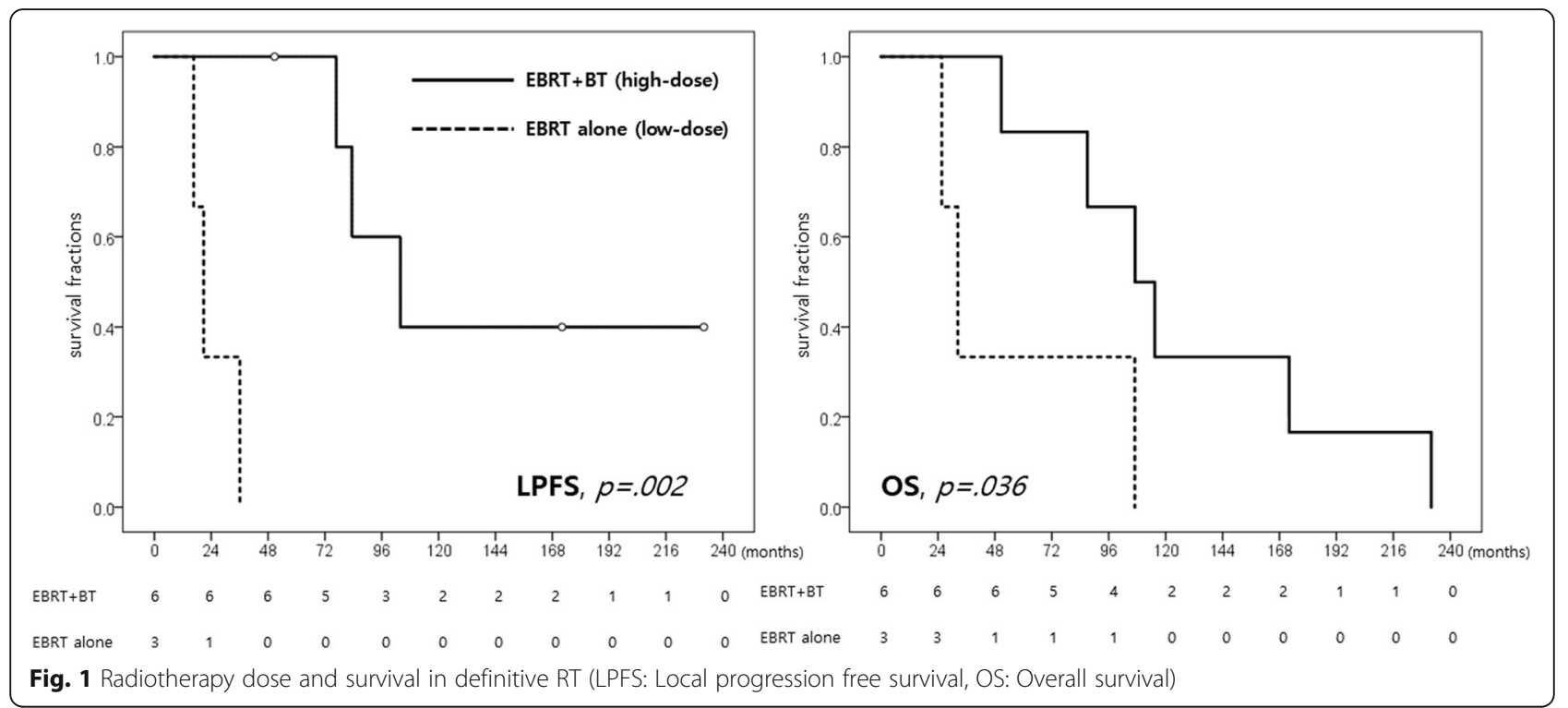

risk of tracheal stenosis should be carefully considered when determining the RT plan or dose in both PORT or definitive RT. Moreover, we should consider a suitable intervention for resolving the symptoms of stenosis, including the growth of granulation tissue [22].

In the current study, we enrolled a small number of patients over a period of 14 years, and analyzed the data retrospectively. However, we followed up patients over a mean period of 10 years and primarily assessed them based on the use of RT. Hence, we could readily determine that patients with unresectable tracheal ACC could survive for a long duration after definitive RT.

In summary, tumor stage and surgical excision might be important factor for survival or local tumor control. However, considering the high possibility of residual disease after surgical resection, postoperative adjuvant RT should be considered to sterilize microscopic tumors after surgery for tracheal ACC. Moreover, unresectable tracheal ACC can effectively be treated with definitive radiotherapy, which yields relatively long-term survival ( $>5$ years) in most patients. Especially in patients with high-dose RT, definitive RT can warrant long-term survival and local tumor control over 5 years with proper methods for beam delivery like IMRT or BT. The major pattern of failure was lung metastasis, regardless of whether surgery was performed. However, tracheal stenosis remains a major concern after RT,

Table 7 Historical reports of treatment for ACC of the trachea

\begin{tabular}{|c|c|c|c|c|c|c|}
\hline Author & Year & $\begin{array}{l}\text { Pt. } \\
\text { No. }\end{array}$ & Treatment & RT dose (Gy) & $\begin{array}{l}\text { 5-yr. OS } \\
(\%)\end{array}$ & Mean OS (months) \\
\hline \multirow[t]{2}{*}{ Grillo [7] } & 1990 & 80 & $S$ & - & - & 39 \\
\hline & & & $S+R$ & NA & - & 107 \\
\hline Gelder [8] & 1993 & 34 & $S(9), S+R(10) R(11), C(4)$ & NA & 80 & - \\
\hline \multirow[t]{2}{*}{ Maziak [6] } & 1996 & 38 & $S(6), S+R(26)$ & NA & 79 & 87 \\
\hline & & & $R(6)$ & $50-75$ & - & 74 \\
\hline Regnard [9] & 1996 & 65 & $S(37), S+R(28)$ & NA & 73 & \\
\hline Prommegger [20] & 1998 & 16 & $S(16)$ & & 79 & - \\
\hline Bhattacharyya [4] & 2004 & 19 & NA & NA & 78 & - \\
\hline Honings [21] & 2010 & 108 & $S(19), S+R(89)$ & 54 & 78 & - \\
\hline \multirow[t]{2}{*}{ Lee [22] } & 2011 & 29 & $S \pm R(17)$ & 54 & 100 & 143 \\
\hline & & & $R(12)$ & 60 & 54 & 61 \\
\hline \multirow[t]{2}{*}{ This study } & 2017 & 22 & $S+R(13)$ & 50 & 92 & 136 \\
\hline & & & $\mathrm{R}(9)$ & 74 & 67 & 128 \\
\hline
\end{tabular}


and the RT plan or dose should accordingly be adjusted in some patients.

\section{Conclusion}

Adjuvant radiotherapy for controlling microscopic residual disease in ACC may be suitable in cases of surgically resected tumors, whereas definitive radiotherapy may yield long-term survival in more than $50 \%$ of patients with unresectable tracheal ACC. Dose escalation should be considered to warrant long-term survival in definitive RT.

\begin{abstract}
Abbreviations
ACC: Adenoid Cystic Carcinoma; BT: Brachytherapy; C: Chemotherapy; CCRT: Concurrent Chemo-Radiotherapy; CR: Complete Remission; CT: Computed Tomography; DFS: Disease Free Survival; DMFS: Distant Metastasis Free Survival; EBRT: External Beam Radiotherapy; Gy: Gray; LP: Local Progression; LPFS: Local Progression Free Survival; mo: months; NA: data not available; OS: Overall Survival; PORT: Postoperative Radiotherapy; PR: Partial Remission; R: Radiation; RO: Complete Resection; R1: Microscopically Incomplete Resection; RESP: Initial response after RT; RT: Radiotherapy; RT-DM: Time interval from RT to DM; RT-LP: Time interval from RT to LP; S: Surgery; SCC: Squamous Cell Carcinoma
\end{abstract}

\section{Acknowledgements}

Not applicable.

\section{Funding}

This research was supported by the Basic Science Research Program through the National Research Foundation of Korea (NRF), funded by the Ministry of Education (NRF-2017R1D1A1B03035167), and a grant from the Korea Health Technology R\&D Project through the Korea Health Industry Development Institute (KHIDI), funded by the Ministry of Health \& Welfare, Republic of Korea (grant number: HI15C0972).

\section{Availability of data and materials}

Not applicable.

\section{Authors' contributions}

HUJ, SYS, and EKC contributed in the study design. GMB created the treatment plans. HUJ, WSC, and SYS contributed in analysis of data and drafting of the manuscript. HUJ, DKK, YHK, SYJ, SSK, and SIP collected patient data. HUJ analyzed the clinical data and was a major contributor in writing the manuscript. SYS provided the final approval of the version to be published. And all authors read and approved the final manuscript.

\section{Ethics approval and consent to participate}

The study was approved by the institutional review board of Asan Medical Center, Seoul, Korea (AMC-IRB, 2012-0470).

\section{Consent for publication}

Not applicable.

\section{Competing interests}

The authors declare that they have no competing interests.

\section{Publisher's Note}

Springer Nature remains neutral with regard to jurisdictional claims in published maps and institutional affiliations.

\section{Author details}

${ }^{1}$ Department of Radiation Oncology, Ulsan University Hospital, University of Ulsan College of Medicine, Ulsan, South Korea. ${ }^{2}$ Department of Radiation Oncology, Asan Medical Center, University of Ulsan College of Medicine, 88, Olympic-ro 43-gil, Songpa-gu, Seoul 05505, South Korea. ${ }^{3}$ Department of Thoracic Surgery, Asan Medical Center, University of Ulsan College of Medicine, Seoul, South Korea. ${ }^{4}$ Asan institute for Life Science, Asan Medical Center, University of Ulsan College of Medicine, Seoul, South Korea. ${ }^{5}$ Department of Medical Health Science, Kangwon National University
Graduate School, Chuncheon, South Korea. ${ }^{6}$ Department of Radiation Oncology, Gangneung Asan Hospital, University of Ulsan College of Medicine, Gangneung, South Korea.

Received: 1 May 2017 Accepted: 15 November 2017

Published online: 04 December 2017

\section{References}

1. Perel MI, Koroleva NS. Primary tumors of the trachea. Philadelphia: Saunders; 1987.

2. Pearson F, Cardoso P, Keshavjee S. Primary tumours of the upper airway. 1st ed. New York: Churchill Livingstone; 1995.

3. Licht PB, Friis S, Pettersson G. Tracheal cancer in Denmark: a nationwide study. Eur J Cardiothorac Surg. 2001;19:339-45.

4. Bhattacharyya N. Contemporary staging and prognosis for primary tracheal malignancies: a population-based analysis. Otolaryngol Head Neck Surg. 2004;131:639-42.

5. Ladefoged C, Bisgaard C, Petri J. Solitary renal metastasis 23 years after extirpation of a bronchial adenoid cystic carcinoma. Scand J Thorac Cardiovasc Surg. 1984;18:245-8.

6. Maziak DE, Todd TR, Keshavjee SH, Winton TL, Van Nostrand P, Pearson FG. Adenoid cystic carcinoma of the airway: thirty-two-year experience. J Thorac Cardiovasc Surg. 1996;112:1522-31. discussion 31-2

7. Grillo HC, Mathisen DJ. Primary tracheal tumors: treatment and results. Ann Thorac Surg. 1990;49:69-77.

8. Gelder CM, Hetzel MR. Primary tracheal tumours: a national survey. Thorax. 1993;48:688-92.

9. Regnard JF, Fourquier P, Levasseur P. Results and prognostic factors in resections of primary tracheal tumors: a multicenter retrospective study. The French Society of Cardiovascular Surgery. J Thorac Cardiovasc Surg. 1996; 111:808-13. discussion 13-4

10. Kanematsu T, Yohena T, Uehara T, Ushijima C, Asoh H, Yoshino I, et al. Treatment outcome of resected and nonresected primary adenoid cystic carcinoma of the lung. Ann Thorac Cardiovasc Surg. 2002;8:74-7.

11. Hsu AA, Tan EH, Takano AM. Lower respiratory tract adenoid cystic carcinoma: its Management in the Past Decades. Clin Oncol (R Coll Radiol). 2015;27:732-40

12. Muller A, Stockamp B, Schnabel T. Successful primary radiation therapy of adenoid cystic carcinoma of the lung. Oncology. 2000;58:15-7.

13. Allen AM, Rabin MS, Reilly JJ, Mentzer SJ. Unresectable adenoid cystic carcinoma of the trachea treated with chemoradiation. J Clin Oncol. 2007; 25:5521-3.

14. Bonner Millar LP, Stripp D, Cooper JD, Both S, James P, Rengan R. Definitive radiotherapy for unresected adenoid cystic carcinoma of the trachea. Chest. 2012;141:1323-6.

15. Tsuchida $Y$, Therasse $P$. Response evaluation criteria in solid tumors (RECIST): new guidelines. Med Pediatr Oncol. 2001;37:1-3.

16. Gaissert HA, Mark EJ. Tracheobronchial gland tumors. Cancer Control. 2006; 13:286-94.

17. Gaissert HA, Grillo HC, Shadmehr MB, Wright CD, Gokhale M, Wain JC, et al. Long-term survival after resection of primary adenoid cystic and squamous cell carcinoma of the trachea and carina. Ann Thorac Surg. 2004;78:1889-96. discussion 96-7

18. Azar T, Abdul-Karim FW, Tucker HM. Adenoid cystic carcinoma of the trachea. Laryngoscope. 1998;108:1297-300.

19. Webb BD, Walsh GL, Roberts DB, Sturgis EM. Primary tracheal malignant neoplasms: the University of Texas MD Anderson Cancer Center experience. J Am Coll Surg. 2006;202:237-46.

20. Prommegger R, Salzer GM. Long-term results of surgery for adenoid cystic carcinoma of the trachea and bronchi. Eur J Surg Oncol. 1998;24:440-4.

21. Honings J, Gaissert HA, Weinberg AC, Mark EJ, Wright CD, Wain JC, et al. Prognostic value of pathologic characteristics and resection margins in tracheal adenoid cystic carcinoma. Eur J Cardiothorac Surg. 2010;37:1438-44.

22. Lee JH, Jung EJ, Jeon K, Koh WJ, Suh GY, Chung MP, et al. Treatment outcomes of patients with adenoid cystic carcinoma of the airway. Lung Cancer. 2011:72:244-9. 